\title{
ABSORPTION LINES IN TRANSITION METAL COMPLEXES
}

\author{
IKUJI TsuJikawa \\ Department of Chemistry, Faculty of Science, \\ Kyoto University, Kyoto, Japan
}

\begin{abstract}
For the past sixteen years, the absorption lines in complexes of the iron group have been investigated on the basis of ligand field theory. These studies, and in particular our recent studies involving chromium complexes, are reviewed.
\end{abstract}

\section{INTRODUCTION}

The absorption spectra of complexes of the iron group consist of absorption bands and absorption lines. Whereas extensive studies of the absorption bands have been made since Werner's day, the absorption lines have so far attracted only a little attention and have been studied only by a few individual workers since about 1930. In 1954, the ligand field theory was developed by Tanabe and Sugano ${ }^{1}$, and by $\mathrm{Orgel}^{2}$, based on the crystal field theory of Bethe. Since then, the absorption lines as well as the absorption bands have been investigated from the standpoint of ligand field theory. The optical anisotropy and the Zeeman effect of absorption lines in ruby were successfully analysed in $1958^{3,4}$. Since then, the absorption lines arising from ions of the iron group in solids have been of considerable interest.

Of course, the absorption lines appear to be more capable of resolution than do the absorption bands. This makes it relatively easy to obtain initial splittings, $g$ values, multiplicities etc. of excited states, as well as the intensities of transition components. It also makes it possible to assign excited states and to deduce transition characters, besides leading to an understanding of the detailed character of complexes.

I shall first explain briefly the mechanisms of transitions and review results obtained from experiments, and then survey the studies of absorption lines in chromium complexes in our laboratory. They concern (1) excited states and transition characters from the standpoint of the ligand field theory, (2) thermal behaviour of the absorption lines, and (3) the electric effect of the environment on the absorption lines.

\section{MECHANISMS OF TRANSITIONS}

The level of an electron in the partly filled shell of transition metal complexes, namely that of a single $d$ electron, splits into the $t_{2 g}$ and $e_{g}$ levels in 
the ligand field of cubic symmetry $\mathrm{O}_{h}$. The ligand field parameter $\Delta$ is defined as the energy between these two levels. The partly filled shell represents electronic configurations $t_{2 g}^{n^{\prime}} e_{g}^{n-n^{\prime}}(1 \leqslant n \leqslant 9)$. If the Coulomb interaction is taken into account, $S \Gamma$ multiplets also split, like $S L$ multiplets of $d^{n}$ configurations in the free ion. Here $\Gamma$ represents one of the irreducible representations of $O_{h}$ namely $A_{1 g}, A_{2 g}, E_{g}, T_{1 g}$ or $T_{2 g}$. If angular dependences of the molecular orbitals $t_{2 g}$ and $e_{g}$ are approximated as those of the $d$ orbitals in the free ion, the energies of the multiplets are expressed with the Slater integrals $F_{0}, F_{2}$ and $F_{4}$ or with Racah's parameters $A \equiv F_{0}-49 F_{4}$, $B \equiv F_{2}-5 F_{4}$ and $C \equiv 35 F_{4} . F_{0}$ or $A$ is independent of the relative energies of the multiplets and the relation $C \approx 4 B$ holds. It follows immediately that the relative energies of the multiplets can be expressed only with $\Delta$ and $B$. The absorption bands correspond to transitions in which one electron is promoted from the $t_{2 g}$ orbital to the $e_{g}$ one, expressed as $t_{2 g}^{n^{\prime}} e_{g}^{n-n^{\prime}} S \Gamma \rightarrow$ $t_{2 g}^{n^{\prime}-1} e_{g}^{n-n^{\prime}+1} S \Gamma^{\prime}$ and $\Delta$ contributes to transition energies ${ }^{1} . \Delta$ has a width of about $3000 \mathrm{~cm}^{-1}$ due to the zero point vibration of a complex. On the other hand, the absorption lines correspond to transitions between states with the same electronic configurations but with total spins different by one unit, expressed as $t_{2 g}^{n} e_{g}^{n-n^{\prime}} S \Gamma \rightarrow t_{2 g}^{n} e_{g}^{n-n^{\prime}} S \pm 1 \Gamma^{\prime}$. There is no electronic excitation $t_{2 g} \rightarrow e_{g}$, so that $\Delta$ does not contribute to the transition energies. The absorption lines are spin forbidden, because the transition moment of an electric dipole or of a magnetic dipole has no matrix elements between states with different spin multiplicities. In addition, absorption lines as well as the absorption bands, are forbidden by the Laporte rule, because states from $d^{n}$ configurations have even parity. Hence the absorption lines are doubly forbidden by spin and parity.

The spin orbit interaction $V_{\mathrm{so}}$ is required to mix states with different spin multiplicities. It leads to the situation that spin forbidden transitions are to be allowed. The spin orbit interaction has matrix elements between states with total spins differing by one unit. The wavefunction for a state $\left(\alpha_{0} \Gamma_{T}\right)$ is

$$
\Phi\left(\alpha_{o} \Gamma_{T}\right)=a \Phi_{0}\left(\alpha S \Gamma, \Gamma_{T}\right)+\sum_{i} b_{i} \Phi_{i}\left(\alpha^{\prime} S \pm 1 \Gamma^{\prime}, \Gamma_{T}\right)
$$

where $\Gamma_{T}$ is the irreducible representation of $S \times \Gamma$. Equation 1 shows that states with the same $\Gamma_{T}$ mix only with each other. The coefficient $b_{i}$ is given by perturbation theory as

$$
b_{i}=\frac{\left(\Phi_{i}\left|V_{\mathrm{so}}\right| \Phi_{0}\right)}{E\left(\Phi_{i}\right)-E\left(\Phi_{0}\right)}
$$

Generally speaking, $a$ is nearly equal to 1 , and $b_{i}$ has an order of magnitude of the multiplet width divided by the energy difference between different multiplets. In the case of iron group ions, the multiplet width is about $100 \mathrm{~cm}^{-1}$ and the energy between different multiplets about $3000 \mathrm{~cm}^{-1}$. Then, the $b_{i}$ is crudely estimated as $3 \times 10^{-2}$.

The mechanisms for introducing parity-forbidden transitions are explained below:

(1) There is the electric dipole transition due to orbital deformations by a 
stress of the crystal field, in other words, due to: (a) an odd parity crystal field arising from thermal vibrations of the crystal, $V_{\text {ov }}$. A transition allowed by this mechanism is called a vibronically allowed one; (b) a hemihedral crystal field $V_{\text {hem }}$ in the absence of the inversion centre of the crystal field, still at equilibrium positions of atoms.

2. There is the magnetic dipole transition. The magnetic dipole is an axial vector which has even parity. It follows that transitions between states with even parity are allowed by the magnetic dipole. Similarly, an electric quadrupole transition is also possible but has not been found experimentally.

We shall estimate an order of magnitude of the oscillator strength, $f$. $f$ is related to the absorbent coefficient, $K(v)$

$$
f=\left(m c / \pi N e^{2}\right) \int K(v) \mathrm{d} v
$$

where $N$ is the number of absorption centres per $\mathrm{cm}^{3}$ and $v$ is the frequency.

Now consider the case 1(a). The crystal field $V(\vec{r}, \vec{Q})$ is expanded in a power series of $Q\left(\gamma, \boldsymbol{m}_{\gamma}\right)$. Here $\vec{r}$ is the electronic coordinate and $Q\left(\gamma, m_{\gamma}\right)$ the normal coordinate of the $\gamma m_{\gamma}$-th mode lattice vibration, namely,

$$
V(\vec{r}, \vec{Q})=V_{o}(\vec{r})+\sum_{\gamma m_{\gamma}} V\left(\gamma m_{\gamma}\right) Q\left(\gamma m_{\gamma}\right)+\ldots
$$

and

$$
\vec{r} V\left(\gamma m_{\gamma}\right)=\left(\frac{\partial V}{\partial Q\left(\gamma m_{\gamma}\right)}\right)_{Q\left(\gamma m_{\eta}\right)=0}
$$

where $V_{0}(r)$ is the crystal field at the nuclear equilibrium positions. Now we assume that the $d$ electrons interact strongly only with vibrations of the complex ion. The second term of equation 4 mainly contributes to $V_{\mathrm{ov}}$ and then $V_{\mathrm{ov}}$ is given by

$$
V_{\mathrm{ov}}=\sum_{\gamma m_{\gamma}} V\left(\gamma m_{\gamma}\right) Q\left(\gamma m_{\gamma}\right)
$$

where $y$ represents odd type vibrational modes of the complex ion. They are $f_{1 u}^{a}, f_{1 u}^{b}$ and $f_{2 u}$ if we deal with the case of the regular octahedral domplex. The magnitude of $V_{\mathrm{ov}}$ is crudely estimated as follows

$$
V_{\mathrm{ov}} \simeq(\partial R / \bar{r}) V_{0}
$$

where $\partial R$ is the zero point amplitude of vibration, and $\bar{r}$ the mean of $r . \partial R$ is obtained from the relation

$$
2 \pi \mu v^{2}(\partial R)^{2}=\frac{1}{2} h v
$$

where $\mu$ is the effective mass of the complex. If $v$ is taken as $10^{13} \mathrm{~s}^{-1}$ and $\mu$ as $4 \times 10^{-23} \mathrm{~g}, \partial R$ may be estimated as about $10^{-9} \mathrm{~cm}$. If $\bar{r}$ is taken as $10^{-8} \mathrm{~cm}$ and $V_{0}$ as $10^{4} \mathrm{~cm}^{-1}, V_{\mathrm{ov}}$ turns out to about $10^{3} \mathrm{~cm}^{-1}$. An even parity wavefunction $\Phi_{g}$ is contaminated with an odd parity wavefunction $\Phi_{u}$ due to $V_{\mathrm{ov}}$ and then the wavefunction is written including vibrational wavefunctions $\chi_{n}$ amd $\chi_{\bar{n}}$ as

$$
\Phi \chi_{n}=c \Phi_{g} \chi_{n}+\sum_{i} d_{i} \Phi_{u, i} \chi_{\bar{n}}
$$


The coefficient $c$ is commonly nearly equal to 1 and

$$
d_{i}=\left(\Phi_{g} \chi_{n}\left|V_{\mathrm{ov}}\right| \Phi_{u, i} \chi_{\bar{n}}\right) /\left\{E\left(\Phi_{g}\right)-E\left(\Phi_{u, i}\right)\right\}
$$

where $\chi_{n}$ and $\chi_{\bar{n}}$ have quantum numbers differing by only one unit. In the absorption spectra at low temperatures, $\chi_{n}$, is the state with all the quantum numbers equal to 0 in all vibrational modes and $\chi_{\bar{n}}$ is the one with the quantum number promoted to 1 in any one of the odd type vibrational modes. If $V_{\text {ov }}$ is taken as $10^{3} \mathrm{~cm}^{-1}$ and $E\left(\Phi_{u}\right)-E\left(\Phi_{g}\right)$ as $10^{5} \mathrm{~cm}^{-1}$, the degree of contamination, $d$ will be about $10^{-2}$. The oscillator strength of the allowed electric dipole transition, $f_{\text {allow }}$, is expressed as

$$
f_{\text {allow }} \simeq\left(8 \pi^{2} m / 3 h\right) v \bar{r}^{2}
$$

and its order of magnitude is 1 . Therefore $f$ in this case, $f_{\text {elec }}$, is given by

$$
f_{\text {elec }}=b^{2} \times d^{2} \times f_{\text {allow }} \simeq 10^{-7}
$$

In the absorption band $b^{2}$ in equation 1 can be omitted and it follows that $f$ is estimated as about $10^{-4}$.

In the case 1(b) the first term of equation 1 can be considered as

$$
V_{0}(\vec{r})=V_{\text {hol }}(\vec{r})+V_{\text {hem }}(\vec{r})
$$

where $V_{\text {hol }}$ is the even parity crystal field which causes states to split as already mentioned. $V_{\text {hem }}$ does not contribute to splittings between states, nor to the thermal and magnetic properties of the substance. Hence, it is difficult to estimate its magnitude empirically, but roughly speaking $V_{\text {hem }}$ might have the same order of magnitude as $V_{\mathrm{ov}}$. Thus in this case, the oscillator strength might have the order of magnitude of $f_{\text {elec }}$ obtained in equation 12.

The oseillator strength in the case $2: f_{\text {mag }}$ is given by

$$
f_{\text {mag }}=b^{2} \times\left(8 \pi^{2} m / 3 h e^{2}\right) v M^{2} \simeq 4 \times 10^{-9}
$$

where $M$ is the matrix element of the magnetic dipole between the ground and excited states and its magnitude is about one Bohr magneton.

The estimation of oscillator strengths outlined above is so crude that an error by a factor of 10 to $10^{2}$ may enter. However, it is likely that the electric dipole transition is generally more intense than the magnetic dipole one.

\section{INFORMATION OBTAINED FROM SPECTROSCOPIC MEASUREMENTS}

\section{(a) Absorption spectrum}

Transition energies, absorption intensities and absorption widths are obtained from measurements of absorption spectra. Generally, absorption lines shift to shorter wavelengths and become sharp with decreasing temperature. It is desirable to measure absorption lines at low temperatures for better resolution.

\section{(b) Optical anisotropy}

Optical anisotropy exists in all crystals except those of the cubic system. Whether transitions are caused by the electric dipole or by the magnetic 


\section{ABSORPTION LINES IN TRANSITION METAL COMPLEXES}

dipole can be experimentally determined without any theoretical analysis. The case of uniaxial crystals will be explained.

As shown in Figure 1, let incident linearly polarized light be parallel and perpendicular to the optic axis. If we neglect a small anisotropy on the plane perpendicular to the optic axis, cases (a) and (b) give the same situation that both the electric and magnetic vectors of the incident light, i.e. $\vec{E}$ and $\vec{H}$ are perpendicular to the optic axis. In cases (c) and (d), one of $E$ and $H$ is perpendicular and the other parallel to the optic axis. Now measure the absorption lines with the polarized light. If the same spectrum is obtained in cases (a) and (c) [or in the cases (a) and (d)] where $\vec{E}$ (or $\vec{H}$ ) is commonly perpendicular to the optic axis, transitions can be regarded as the electric dipole (or magnetic dipole) transitions.
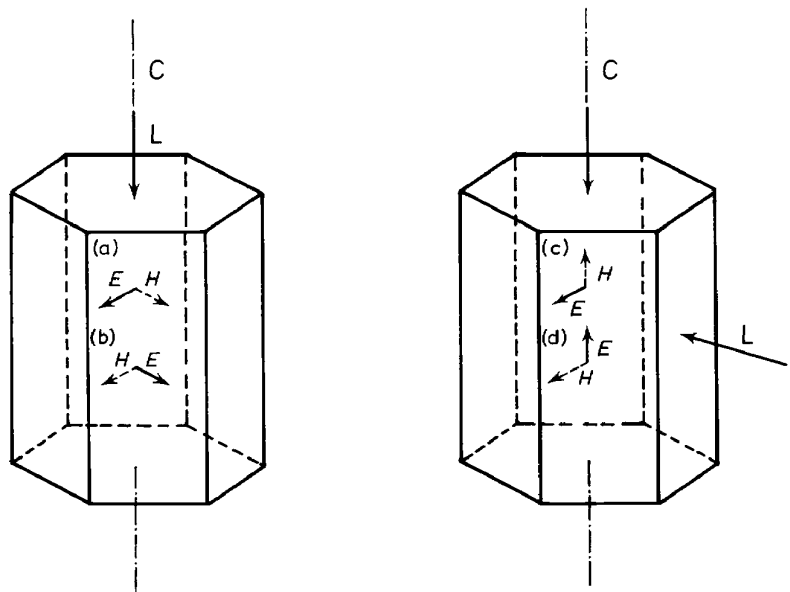

Figure 1. Relation between the optic axis, $C$, the incident linearly polarized light, $L$, the electric vector of the incident light, $\vec{E}$, and its magnetic vector, $\vec{H}$.

\section{(c) The Zeeman effect}

The $g$ values and multiplicities of excited states and intensities of Zeeman components are obtained. In the case of the cubic system, it can be determined experimentally from the anisotropy of the Zeeman effect whether transitions are caused by the electric or magnetic dipole. Multiplicities of excited states are determined by measurements of Zeeman effect in the condition $\mu_{\mathrm{B}} \mathrm{H}_{0}>$ $k T$ where $H_{0}$ denotes a magnetic field. An example is shown in Figure 2. We assume that $g$ values of both the ground and excited states are 2.0 and that the ground state is a quartet. In the condition $\mu_{\mathrm{B}} H_{0}>k T$, populations on higher Zeeman sublevels of the ground state are much smaller than those on lower ones, according to the Bolzmann distribution law. If the excited state is also a quartet, the intensity of the centre Zeeman component does not decrease. On the contrary, if the excited state is a doublet, the intensity of the centre Zeeman component will decrease considerably.

The above is what can be known from experiments alone; to assign excited states and to obtain information about a lower symmetry crystal field and 

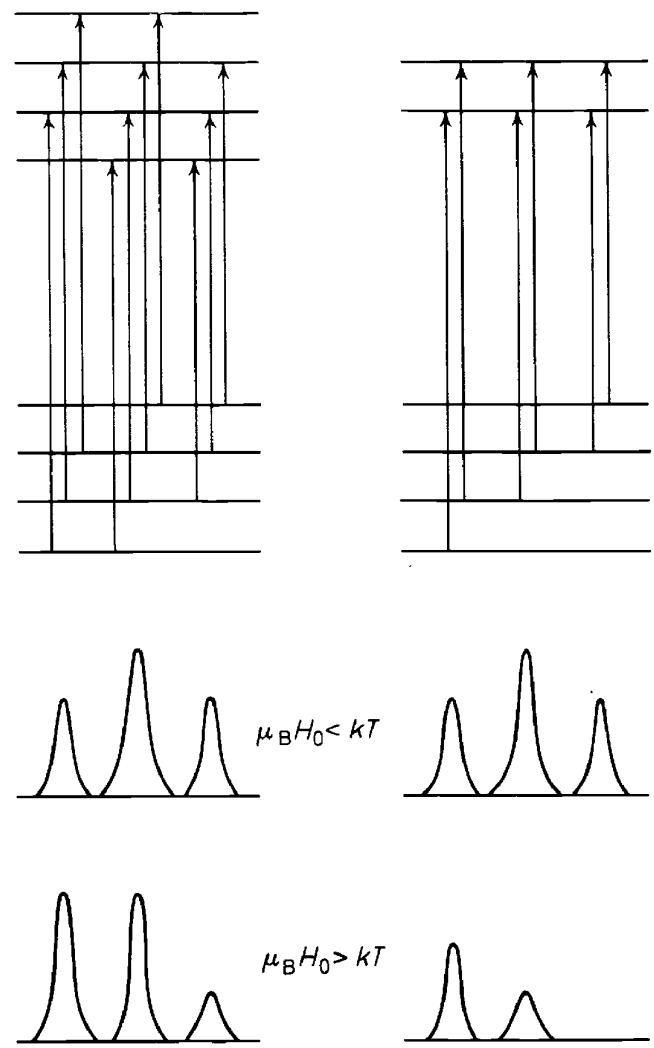

Figure 2. Transition diagram of Zeeman effect and model diagram of Zeeman pattern.

the spin orbit coupling, theoretical analyses are required on the intensities, anisotropies, Zeeman effect etc. In the case of absorption bands, however, it might be difficult to obtain these details due to their lower resolution.

\section{RESEARCH ON EXCITED STATES AND TRANSITION CHARACTERS}

The main iron group octahedral complexes which have been studied are reviewed in Table 1. The ruby $\mathrm{Al}_{2} \mathrm{O}_{3}: \mathrm{Cr}^{3+}, \mathrm{MgO}: \mathrm{Cr}^{3+}$ and $\mathrm{Al}_{2} \mathrm{O}_{3}: \mathrm{V}^{3+}$ are not complexes, but analyses of absorption lines in them were successful on the basis of ligand field theory, so they are shown here together. Mention should be made of the studies of tetrahedral cobaltous compounds by Pelletier-Allard ${ }^{34}$ and by Ferguson ${ }^{35}$. Complexes of the $\mathrm{Cr}^{3+}$ and $\mathrm{Mn}^{2+}$ ions were widely investigated because of absorption widths smaller than $5 \mathrm{~cm}^{-1}$. Complexes of the $\mathrm{Cr}^{2+}, \mathrm{Fe}^{3+}$ and $\mathrm{Fe}^{2+}$ ions can be expected to have absorption lines in view of their electronic configurations, but they have been little studied.

Studies of absorption lines in iron group complexes have been limited 


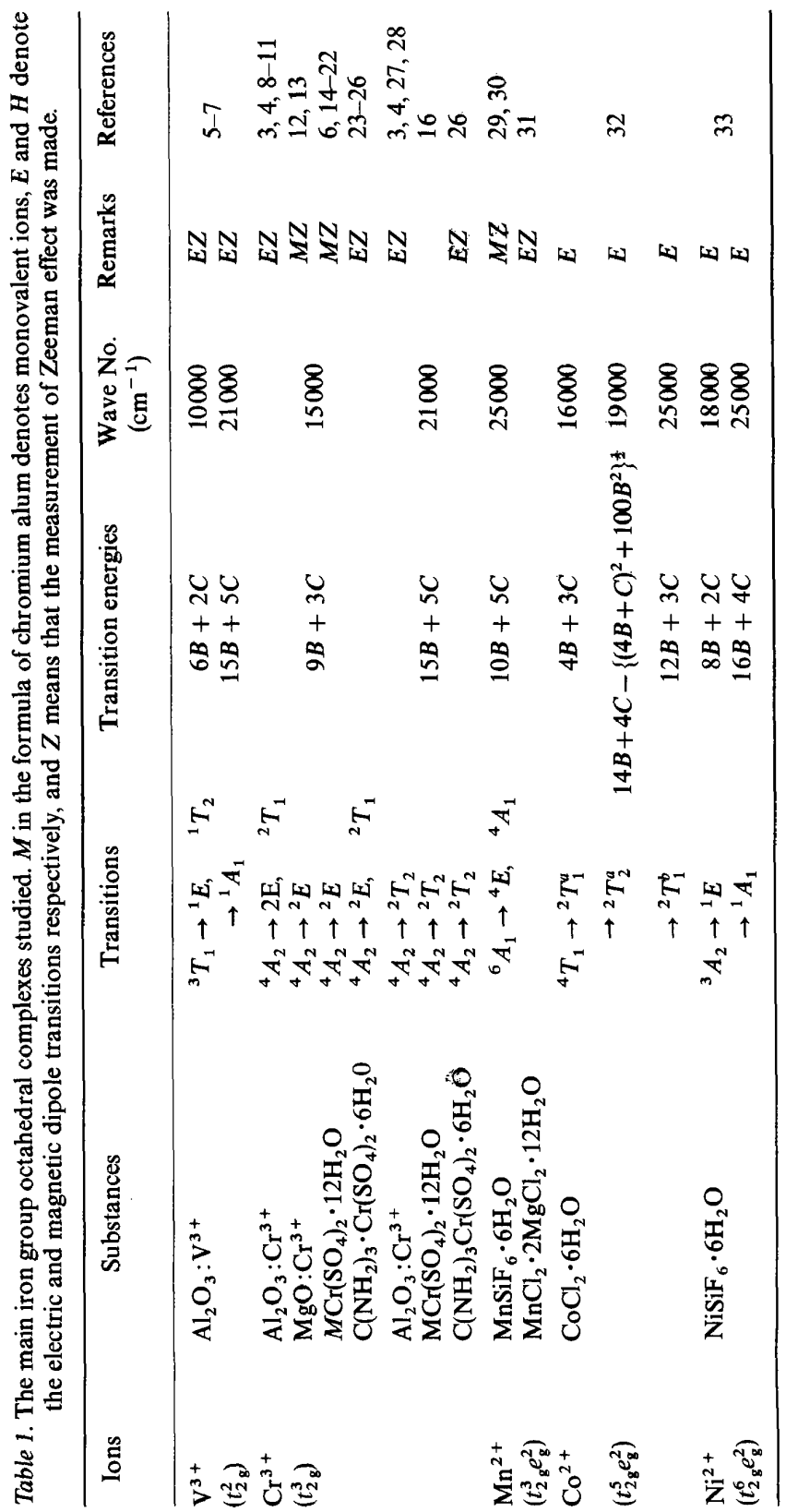


owing to several experimental restrictions: (1) Crystals of sufficient dimension are needed to permit measurement. (2) Crystals must maintain transparency without any damage at low temperatures. (3) Higher magnetic fields than usual $(30 \mathrm{kOe})$ are required to measure the Zeeman effect of absorption lines of widths larger than $2 \mathrm{~cm}^{-1}$. (4) It may happen that liquid helium or hydrogen, a strong magnetic field and a high resolution spectrograph are all needed in the same experiment.

\section{(a) Thallium chromium alum, $\mathrm{TlCr}\left(\mathrm{SO}_{4}\right)_{2} \cdot 12 \mathrm{H}_{2} \mathrm{O}$}

Alums belong to the cubic system and the unit cell contains four molecules. Each $\mathrm{Cr}^{3+}$ ion is surrounded by six water molecules with a nearly regular octahedral arrangement. The $\mathrm{Cr}^{3+}$ ions are placed along the four body diagonals of the unit cell and exposed to the crystal field with the trigonal symmetry $C_{3 i}$. Thallium chromium alum has two absorption lines, $R_{1}: 14943$ $\mathrm{cm}^{-1}$ and $R_{2}: 14945 \mathrm{~cm}^{-1}$. Measurements of absorption lines were made photographically by use of a grating spectrograph with a dispersion of $2.5 \AA / \mathrm{mm}$ in the first order at low temperatures down to $1.3^{\circ} \mathrm{K}$. For the measurement of the Zeeman effect, a magnetic field of $27 \mathrm{kOe}$ was applied parallel to the cubic axis $C_{2}$, the body diagonal $C_{3}$ and face diagonal $C_{0}$ of the crystal. The results are shown in Figure 3. Anisotropy, not observed in the absence of magnetic field, is produced by applying the magnetic field. Zeeman components of the longer wavelength side almost disappear at $1.3^{\circ} \mathrm{K}$ because of the reason already mentioned. Figure 4 shows the Zeeman effect with three directions of the incident light $L$ by use of the magnetic field parallel to the $C_{0}$ axis. The three spectra with the same direction of $H$ but with different direction of $E$ are identical. It follows immediately that the transitions are caused by the magnetic dipole. This is reasonable because the site symmetry of the $\mathrm{Cr}^{3+}$ ion has an inversion centre.

Now we shall analyse the $g$ tensors of the excited states and Zeeman patterns of the $R$ lines, and give predicted Zeeman patterns. The Hamiltonian for the $\mathrm{Cr}^{3+}$ ion in chromium alum is given by

$$
H=H_{0}+H^{\prime}
$$

and

$$
H^{\prime}=V_{\text {trig }}+V_{\text {so }}
$$

where $H_{0}$ is the Hamiltonian for the cubic field, $V_{\text {trig }}$ the trigonal field and $V_{\text {so }}$ the spin-orbit interaction. Here $H^{\prime}$ may be considered as a small perturbation. The group theory, in which both the crystal symmetry and the time-reversal symmetry are taken into account, leads to the effective Hamiltonian for the excited state ${ }^{2} E$ as follows.

$$
H_{\text {eff }}=\lambda S_{z} T\left(A_{2}\right)
$$

where $\lambda$ is a parameter to be determined empirically and $T\left(A_{2}\right)$ is an orbital 


\section{ABSORPTION LINES IN TRANSITION METAL COMPLEXES}

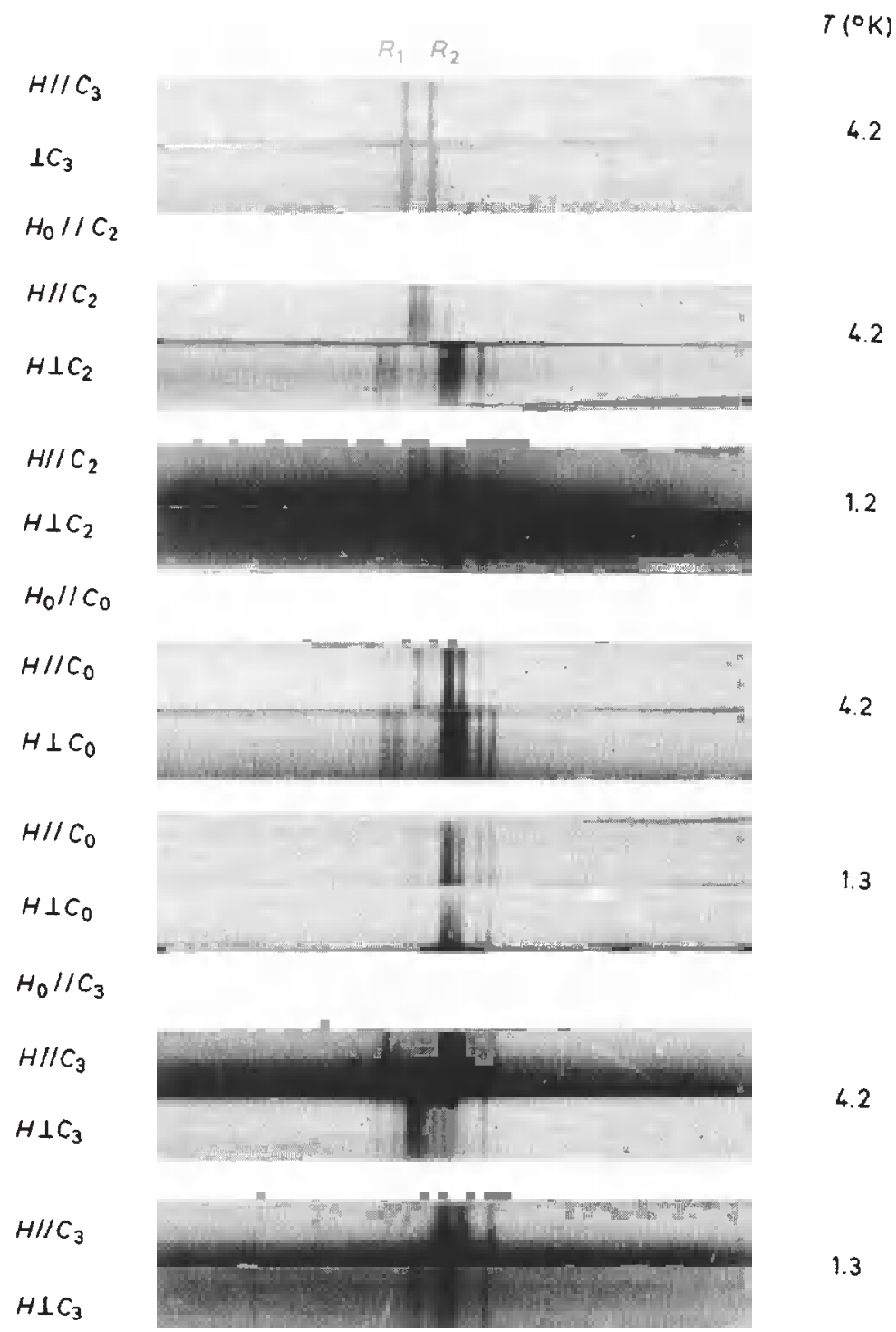

Figure 3. Zeeman patterns of the $R$ lines for various directions of the magnetic field at liquid helium temperatures in thallium chromium alum. 
IKUJI TSUJIKAWA

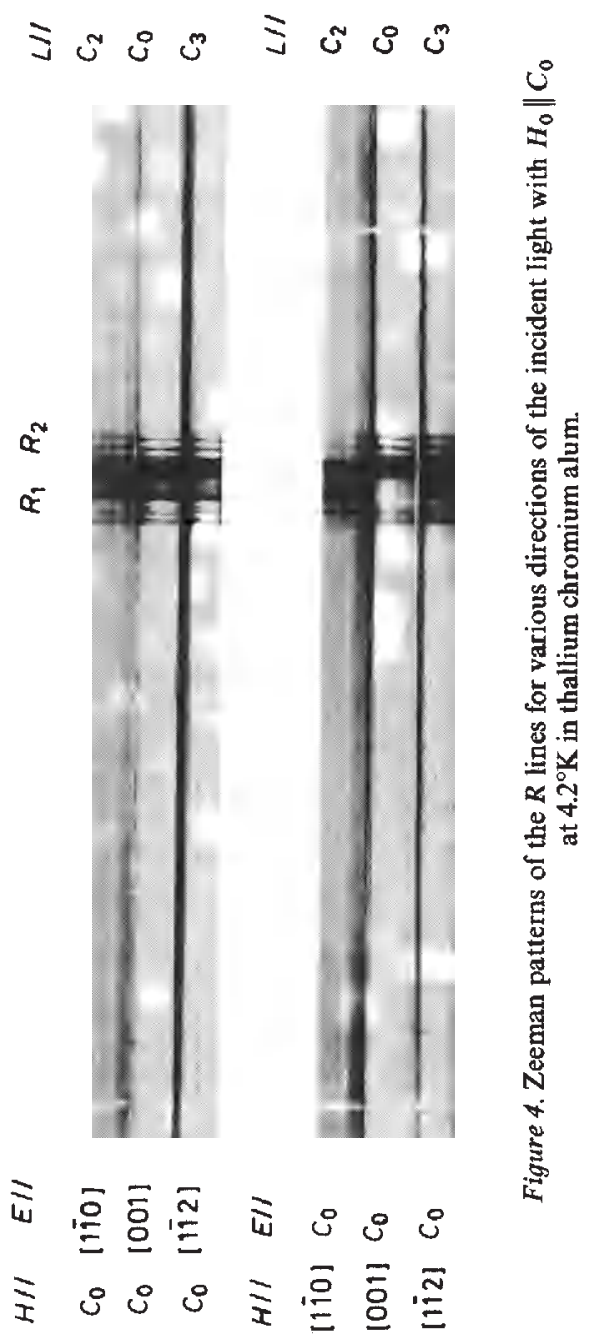


ABSORPTION LINES IN TRANSITION METAL COMPLEXES

operator. The eigenvalues and corresponding eigenfunctions are:

$$
\begin{aligned}
& E_{2}=\frac{1}{2} \lambda, \quad\left\{\begin{array}{l}
2 \bar{A} \begin{array}{l}
\Phi\left({ }^{2} E, \frac{1}{2} u_{+}\right) \\
\Phi\left({ }^{2} E,-\frac{1}{2} u_{-}\right)
\end{array}
\end{array}\right. \\
& E_{1}=-\frac{1}{2} \lambda,\left\{\begin{array}{l}
\bar{E} \begin{array}{l}
\Phi\left({ }^{2} E, \frac{1}{2} u_{-}\right) \\
\Phi\left({ }^{2} E,-\frac{1}{2} u_{+}\right)
\end{array}
\end{array}\right.
\end{aligned}
$$

where $2 \bar{A}$ and $\bar{E}$ are the two-valued representations of the $C_{3 i}$. According to the second order perturbation in $H^{\prime}$ in which the intermediate states are restricted to states in the configuration $t_{2 g}^{3}$, we find

$$
\lambda\left({ }^{2} E\right)=4 K \zeta /\left\{E\left({ }^{2} E\right)-E\left({ }^{2} T_{2}\right)\right\}
$$

where

$$
K=\left\langle t_{2} x_{ \pm}\left|V_{\text {trig }}\right| t_{2} x_{ \pm}\right\rangle
$$

and

$$
\zeta=-2\left\langle t_{2} \frac{1}{2} x_{+}\left|V_{\mathrm{so}}\right| t_{2} \frac{1}{2} x_{+}\right\rangle
$$

Equation 19 leads to the trigonal field parameter, $K=-25 \mathrm{~cm}^{-1}$, where the spin-orbit coupling parameter, $\zeta=140 \mathrm{~cm}^{-1}$ and $E\left({ }^{2} E\right)-E\left(2 T_{2}\right)=$ $-7000 \mathrm{~cm}^{-1}$ are used.

In order to assign the final states of the transition, the magnetic dipole strengths are calculated for the transitions from the ground state ${ }^{4} A_{2}$ to the Kramers doublets $2 \bar{A}$ and $\bar{E}$ of the ${ }^{2} E$ state. The magnetic dipole strength polarized in $\gamma_{0}$ is given by

$$
\begin{aligned}
S_{\mathrm{mag}}\left({ }^{4} A_{2} M_{s} e_{2} \rightarrow{ }^{2} E \bar{M}_{s} \bar{M}: \gamma_{0}\right) \\
\quad=\frac{\mu_{\mathrm{B}}^{2}\left|\sum_{M}\left({ }^{4} A_{2} M_{s} e_{2}\left|M\left(\gamma_{0}\right)\right|^{4} T_{2} M_{s} M\right)\left({ }^{4} T_{2} M_{s} M\left|V_{\mathrm{so}}\right|^{2} E \bar{M}_{s} \bar{M}\right)\right|^{2}}{\left[E\left({ }^{4} T_{2}\right)-E\left({ }^{2} E\right)\right]^{2}}
\end{aligned}
$$

where $\vec{M}$ is $-\Sigma_{i}\left(l_{i}+2 S_{i}\right)$ and $\gamma_{0}$ is a component of $\vec{M}$.

The angular dependence of $g$ values of the $\bar{E}$ and $2 \bar{A}$ states is shown in Figure 5. If we ignore the difference between the $g$ values of the $\bar{E}$ and $2 \bar{A}$ states, the angular dependence of $g$ values becomes

$$
g\left(R_{1}\right)=g\left(R_{2}\right)=\left[\left(\lambda+g_{\|} \mu_{\mathrm{B}} H_{\mathrm{oz}}\right)^{2}+\left(g_{\perp} \mu_{\mathrm{B}} H_{\mathrm{ox}}\right)^{2}\right]^{\frac{1}{2}} / \mu_{\mathrm{B}} H_{0}
$$

The calculated angular dependence agrees well with experimental results.

The experimental and calculated Zeeman patterns in the case of $H_{0} \| C_{3}$ are shown in Figure 6 . The calculated Zeeman patterns with $\lambda>0$ agree with the experimental ones. It follows immediately that the $R_{1}$ and $R_{2}$ lines correspond to transitions to the $\bar{E}\left({ }^{2} E\right)$ and $2 \bar{A}\left({ }^{2} E\right)$ states respectively.

When a magnetic field is applied along directions other than the trigonal axis, a contamination of spin components occurs through the perpendicular Zeeman terms. If the Zeeman energy exceeds the anisotropy energy, the crystal Paschen-Back effect occurs and the quantization axis of the excited state deviates from the trigonal axis. The experimental and calculated Zeeman 
IKUJI TSUJIKAWA

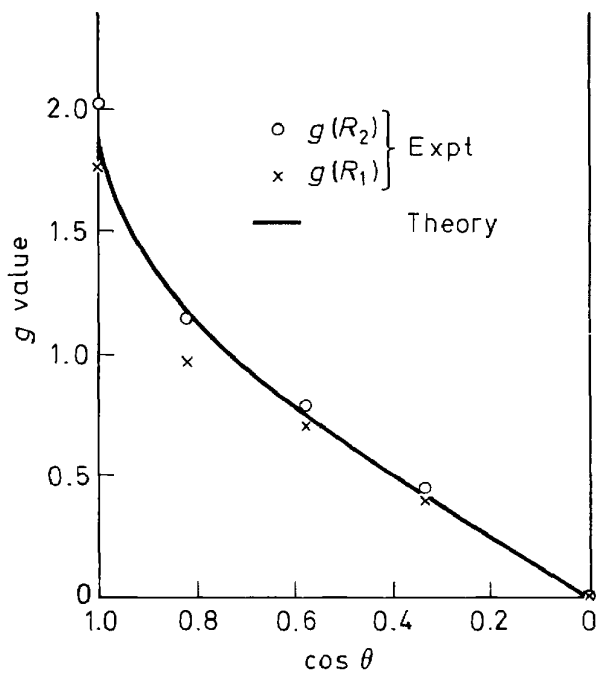

Figure 5. The angular dependence of $g$ values of the excited states which are final levels of the transitions of the $R_{1}$ and $R_{2}$ lines in thallium chromium alum. $\theta$ is the angle between the direction of the magnetic field and the trigonal axis. The theoretical curves are set by using equation 23 , with $\lambda=2.0 \mathrm{~cm}^{-1}, g_{\|}=1.9$ and $g_{\perp}=1.6$
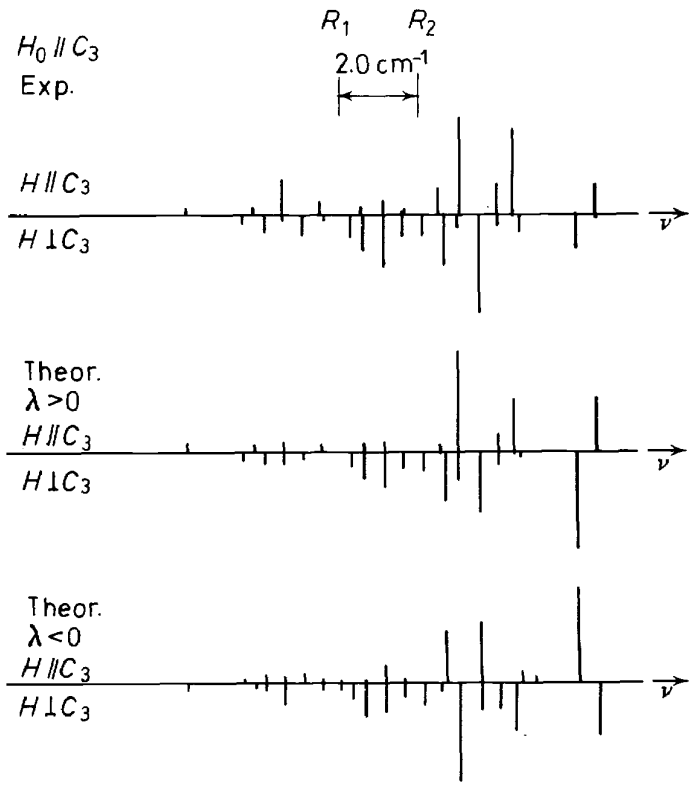

Figure 6. Schematic representations of the experimental and calculated Zeeman patterns of the $R$ lines with $H_{0} \| C_{3}$ at $4.2^{\circ} \mathrm{K}$ in thallium chromium alum. The heights of the solid lines in the patterns represent the relative intensities of the Zeeman components. 


\section{ABSORPTION LINES IN TRANSITION METAL COMPLEXES}

patterns in the case of $H_{0} \| C_{2}$ are shown in Figure 7 . In the middle pattern, the crystal Paschen-Back effect is ignored, i.e. it is assumed that $|\lambda| \gg$ $\left|g_{\perp} \mu_{\mathrm{B}} H_{0}\right|$. By taking into account the Paschen-Back effect, the agreement between the experimental and calculated Zeeman patterns becomes good.
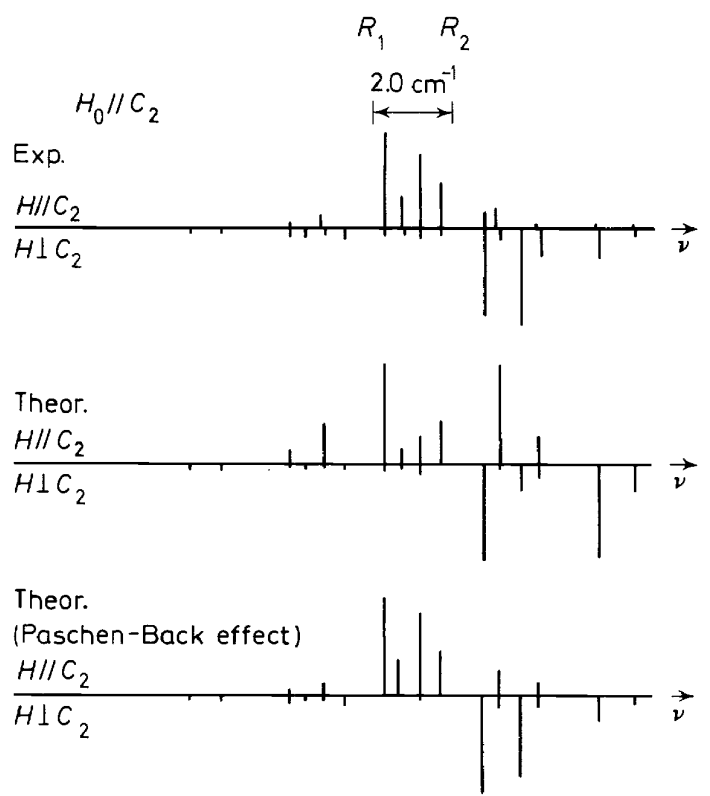

Figure 7. Schematic representations of the experimental and calculated Zeeman patterns of the $R$ lines with $H_{0} \| C_{2}$ at $4.2^{\circ} \mathrm{K}$ in thallium chromium alum.

The Paschen-Back limit pattern could be photographed with a spectrograph with the dispersion of $15 \AA / \mathrm{mm}$ by use of the pulsed magnetic field of $220 \mathrm{kOe}$ as shown in Figure 8 . The $R_{1}$ and $R_{2}$ lines cannot be resolved in the absence of magnetic field due to the low resolution of the spectrograph. The side components shift linearly with the field strength, and the effective splitting factor for the side components is isotropic and about four. When the Zeeman energy exceeds the anisotropy energy of the excited state spin, the excited state spin is quantized to the direction of the magnetic field as well as ground state spin, therefore the nearly degenerate excited states are expected to behave as a free spin doublet. The calculated Paschen-Back patterns with the experimental ones are shown in Figure 9. The agreement between them is good. Then it is ascertained that the $R_{1}$ and $R_{2}$ lines correspond to the magnetic dipole transition from the ground state ${ }^{4} A_{2}$ to the excited state ${ }^{2} E$.

\section{(b) Caesium chromium alum $\mathrm{CsCr}\left(\mathrm{SO}_{4}\right)_{2} \cdot 12 \mathrm{H}_{2} \mathrm{O}$}

Caesium chromium alum has a single absorption line $\left(R: 14479 \mathrm{~cm}^{-1}\right)$ in the red region. A study of the $R$ line was made in a similar way to that of 


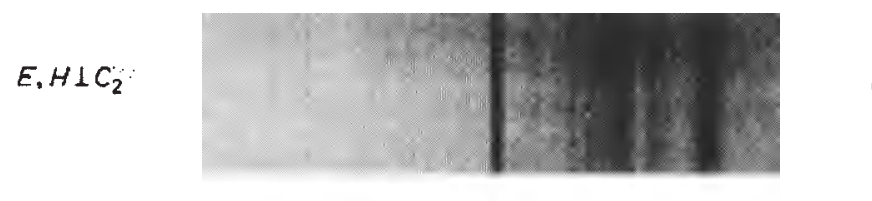

0
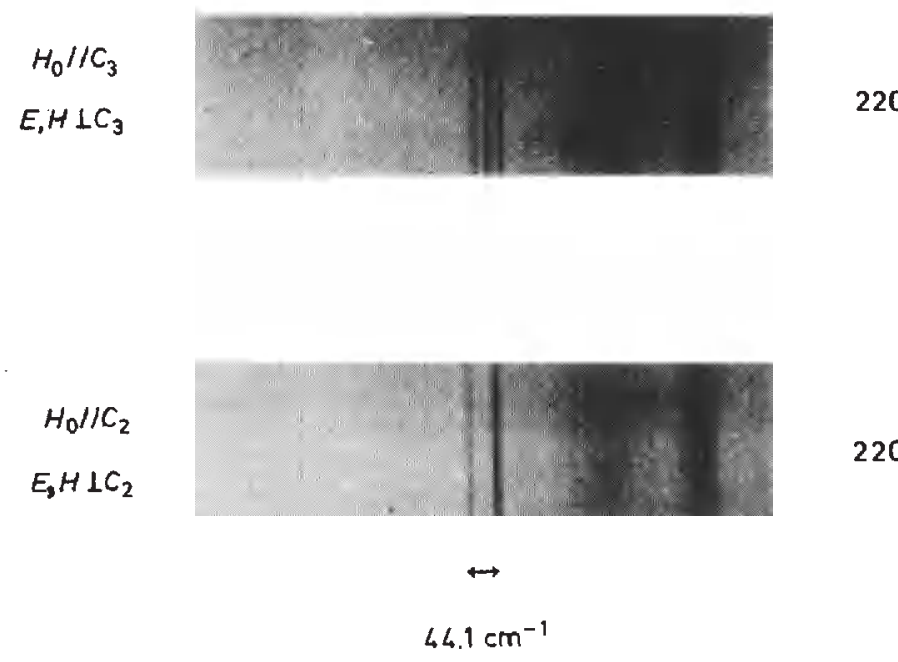

220

Figure 8. Paschen-Back limit patterns formed by applying the pulsed magnetic field of $220 \mathrm{kOe}$ with $\mathrm{H}_{0} \| \mathrm{C}_{2}$ and with $\mathrm{H}_{\mathrm{o}} \| \mathrm{C}_{3}$ at $77^{\circ} \mathrm{K}$ in thallium chromium alum.

thallium chromium alum. Anisotropic Zeeman patterns indicate that the $R$ line corresponds to the transition caused by the magnetic dipole. The experimental and calculated Zeeman patterns are shown in Figures 10 and 11 . In the case of $H_{0} \| C_{2}$, the calculated patterns for the transition to the $\bar{E}\left({ }^{2} E\right)$ state coincide with those for the transition to the $2 \bar{A}\left({ }^{2} E\right)$ state and they agree with the experimental patterns. Other calculated patterns obtained, assuming that a final state is a split component of the ${ }^{2} T_{1}$ state do not agree with the experimental patterns. In the case of $H_{0} \| C_{3}$, the calculated Zeeman patterns for the transition to the $\bar{E}\left({ }^{2} E\right)$ state are different from those for the transition to the $2 \bar{A}\left({ }^{2} E\right)$ state and the former agree well with the experimental patterns. Therefore, the $R$ line may correspond to the transition from the ground state ${ }^{4} A_{2}$ to the excited state $\bar{E}\left({ }^{2} E\right)$. The other transition to the $2 \bar{A}\left({ }^{2} E\right)$ state is not found; the reason is not clear. But the splitting between the $2 \bar{A}$ and $\bar{E}$ states must be much larger than $3.5 \mathrm{~cm}^{-1}$, because the Zeeman components split linearly and the $g$ values of the $\bar{E}$ state are anisotropic with magnetic fields up to $27 \mathrm{kOe}$. 


\section{ABSORPTION LINES IN TRANSITION METAL COMPLEXES}

$$
\begin{array}{ll}
H_{0} / / C_{2} & \\
\text { Exp. } & \text { Theor. } \\
& \text { (mag. dipole) }
\end{array}
$$

$$
\begin{aligned}
& \mathrm{H}_{0} / / \mathrm{C}_{3} \\
& \text { Exp. }
\end{aligned}
$$
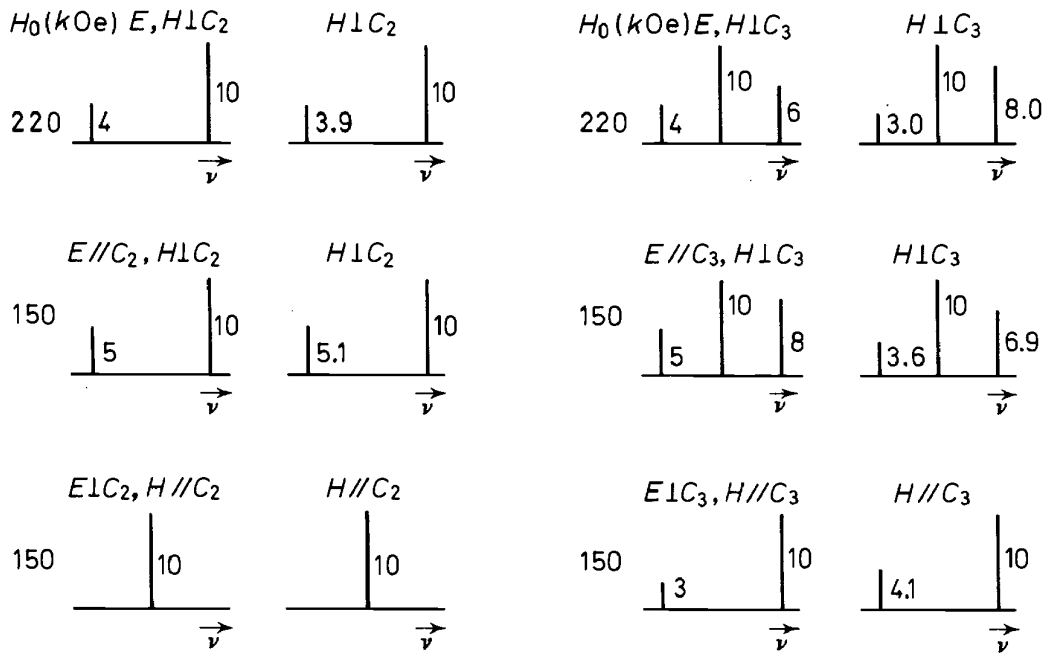
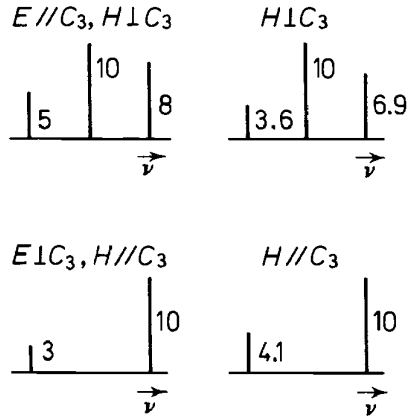

Figure 9. Experimental and theoretical Paschen-Back patterns of the $R$ lines by applying high magnetic fields with $H_{0} \| C_{2}$ and with $H_{0} \| C_{3}$ at $77^{\circ} \mathrm{K}$ in thallium chromium alum.

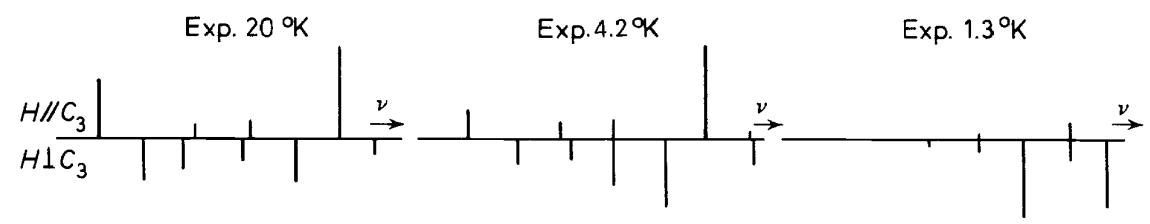

Theor. $2 \bar{A} 20^{\circ} \mathrm{K}$

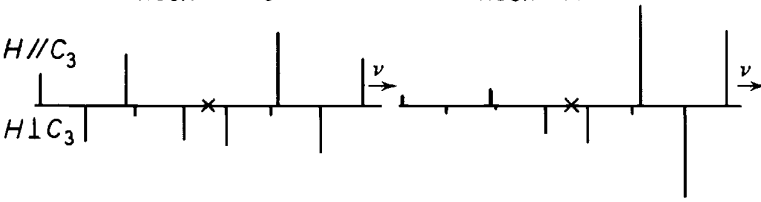

Theor. $\vec{E} 20^{\circ} \mathrm{K}$

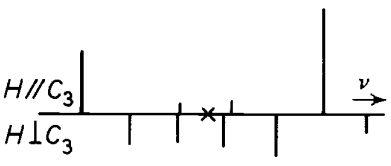

Theor. $\bar{E} 4.2 \%$

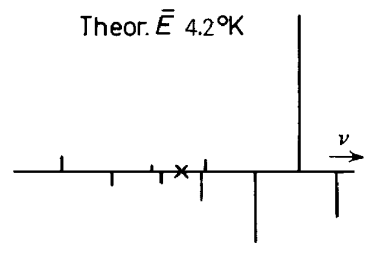

Theor. $2 \bar{A} 1.3^{\circ} \mathrm{K}$

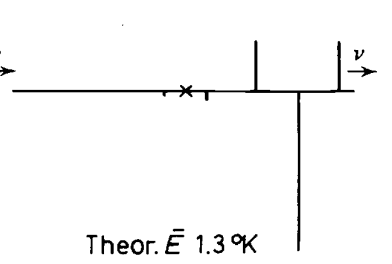

Figure 10. Schematic representations of the experimental and calculated Zeeman patterns of the $R$ line with $H_{0} \| C_{3}$ at $T=20^{\circ} \mathrm{K}, 4.2^{\circ} \mathrm{K}$ and $1.3^{\circ} \mathrm{K}$ in caesium chromium alum 


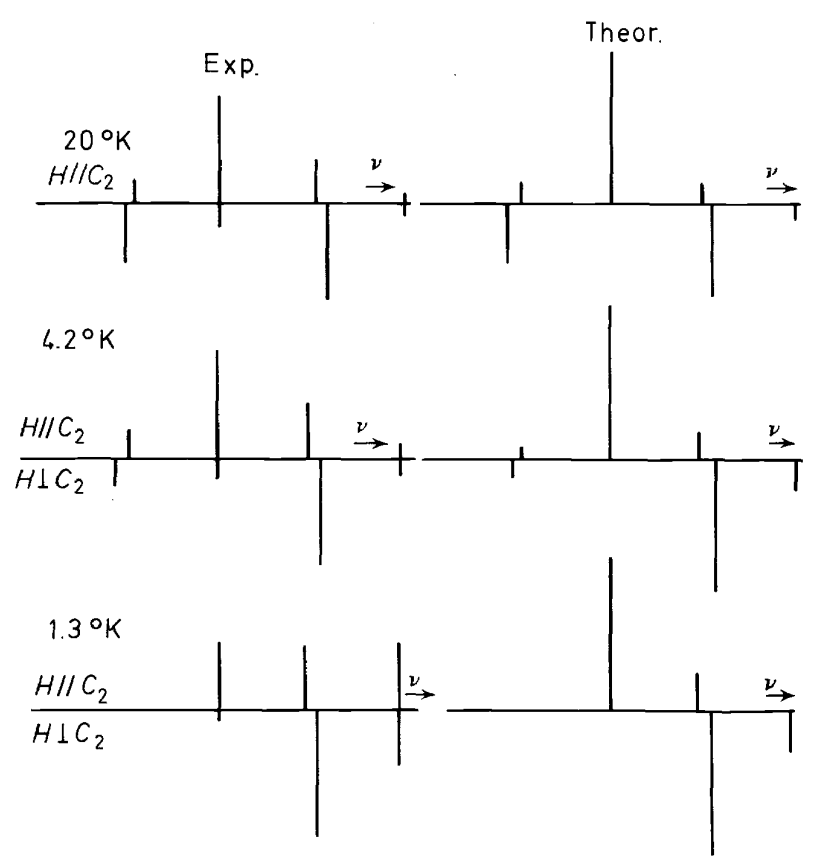

Figure 11. Schematic representations of the experimental and calculated Zeeman patterns of the $R$ line with $H_{0} \| C_{2}$ at $T=20^{\circ} \mathrm{K}, 4.2^{\circ} \mathrm{K}$ and $1.3^{\circ} \mathrm{K}$ in caesium chromium alum.

\section{RESEARCH ON THE THERMAL BROADENING AND THERMAL SHIFT OF ABSORPTION LINES}

The study of the width, shape and position of absorption lines has not advanced in comparison with that of resonance lines of e.s.r. or n.m.r. In the case of iron group complexes, this aspect has not been investigated. We shall discuss the thermal behaviour of the $R$ lines in thallium chromium alum. The magnitude of the magnetic dipole interaction in chromium alum has been estimated to be as small as $10^{-2} \mathrm{~cm}^{-1}$. In fact, the Zeeman patterns of the $R$ lines can be well explained regardless of the magnetic dipole interaction. We might, therefore, at this stage, consider the $\mathrm{Cr}^{3+}$ ions as localized ions in a host crystal and apply a theory of the thermal broadening and shift of zero-phonon lines of impurity ions in a crystal by Di Bartolo ${ }^{36}$.

Thermal broadening mechanisms for sharp zero-phonon lines are classified into the following types. (1) Lifetime broadening due to radiationless decay or excitation of an ion, which involves (a) one phonon process, (b) multi-phonon processes and (c) Raman relaxation or excitation processes. (2) Lifetime broadening due to vibronic decay of an ion from an excited state, which involves one photon emission and one or multi-phonon absorption or emission. (3) Energy change due to adiabatic variation of crystal field, which concerns Raman scattering of phonons by an ion in an excited state. The ion remains in the same electronic state by phonon scattering. The 
temperature dependent width of an ionic level is expressed as follows, in which the Debye phonon state density is assumed.

$$
\begin{aligned}
\Delta W\left(\mathrm{~cm}^{-1}\right)=\alpha\left(\frac{T}{T_{D}}\right)^{7} \int_{0}^{T_{D} T} \frac{x^{6} \mathrm{e}^{x}}{\left(\mathrm{e}^{x}-1\right)^{2}} \mathrm{~d} x & +\frac{1}{C} \sum_{j<i} \beta_{i j}\left(\frac{\exp \left(\Delta E_{i j} / k T\right)}{\exp \left\{\Delta E_{i j} / k T\right\}-1}\right) \\
& +\frac{1}{C} \sum_{j>i} \beta_{i j}\left(\frac{1}{\exp \left\{\Delta E_{i j} / k T\right\}-1}\right)
\end{aligned}
$$

where

$$
\begin{aligned}
\alpha=\frac{1}{C} \frac{9}{2 \pi^{3} \rho^{2} v^{10}}\left(\frac{k T_{D}}{\hbar}\right)^{7} \mid\left\langle\psi_{f}^{\mathrm{e}}\right| & V_{2}\left|\psi_{i}^{\mathrm{el}}\right\rangle \\
& +\left.\sum_{l} \frac{\left\langle\psi_{f}^{\mathrm{el}}\left|V_{1}\right| \psi_{l}^{\mathrm{e} l}\right\rangle\left\langle\psi_{l}^{\mathrm{e}}\left|V_{1}\right| \psi_{i}^{\mathrm{el}}\right\rangle}{E_{i}^{\mathrm{el}}-E_{l}^{\mathrm{el}}}\right|^{2}
\end{aligned}
$$

and

$$
\beta_{i j}=\left.\frac{3 \omega_{i j}^{2}}{2 \pi \rho v^{5} h}\left\langle\psi_{j}^{\mathrm{el}}\left|V_{1}\right| \psi_{j}^{\mathrm{el}}\right\rangle\right|^{2}
$$

where $T_{D}$ is the Debye temperature, $\rho$ the density of crystal, $v$ the mean sound velocity in the crystal, and $V_{1}$ and $V_{2}$ are electron phonon coupling operators. In equation 24 , the first term concerns the Raman processes and the second and third terms concern one phonon excitations and relaxation processes respectively. All the processes in equation 24 contribute to a homogeneous broadening of the linewidth and predict a Lorentzian shape. The energy levels of an ion in the crystal shift with temperatures through the ion-lattice vibration interaction. The shift can be formulated in a similar form as the width of an ionic level.

The $R$ lines were measured photoelectronically with a 'Spex Model 1800' spectrometer at temperatures between $77^{\circ} \mathrm{K}$ and $300^{\circ} \mathrm{K}$. In Figures 12 and 13 , experimental temperature dependences of the linewidth and of the shift are shown together with the calculated ones, and parameters are given for the best fit with experiments. The observed linewidth and shift are strongly dependent on temperature in the range between $77^{\circ} \mathrm{K}$ and $260^{\circ} \mathrm{K}$ respectively. These results are consistent with the two phonon Raman process model having the Debye temperature $120^{\circ} \mathrm{K}$, which is not so far from $T_{D}=80^{\circ} \mathrm{K}$ obtained from a specific heat measurement. The coupling parameter $\beta_{i j}$ is zero, meaning that the one phonon direct processes do not contribute to the linewidth. One of the reasons for $\beta_{i j}=0$ is the small number of available phonons for a single phonon decay. The second reason is the weak electron-phonon coupling $\left\langle\psi_{i}^{\mathrm{el}}\left|V_{1}\right| \psi_{j}^{\mathrm{el}}\right\rangle$, since wavefunctions of the two electronic states $\psi_{i}^{\text {el }}$ and $\psi_{j}^{\text {el }}$ corresponding to the Kramers doublets of the ${ }^{2} E$ state, are almost the same in a dominantly cubic crystal field. The 
IKUJI TSUJIKAWA

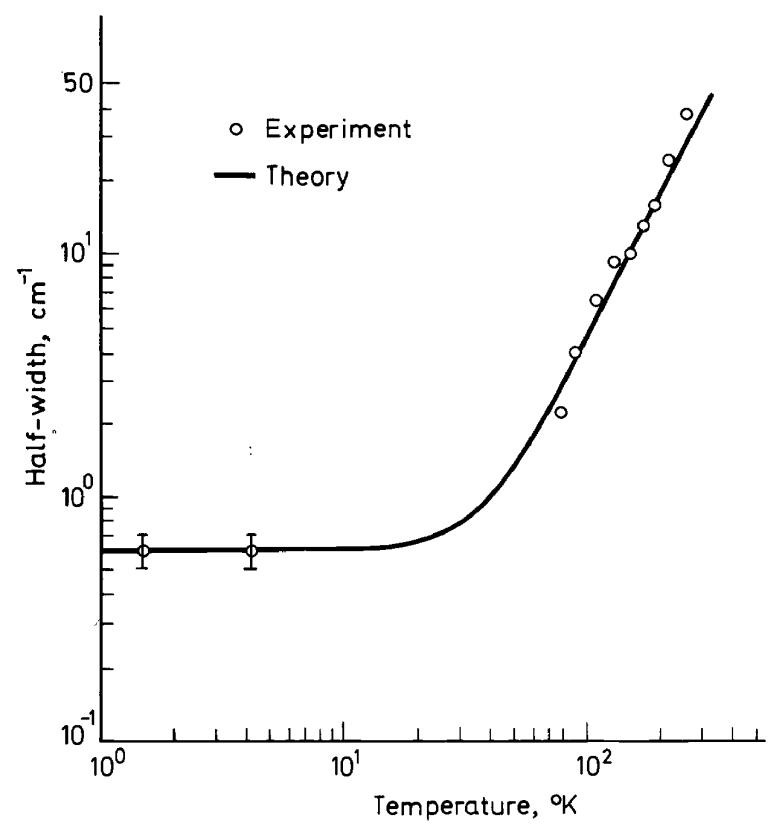

Figure 12. The temperature dependence of the halfwidth of the $R_{1}$ line under the assumption that those of the $R_{1}$ and $R_{2}$ lines are to show the same thermal broadening in thallium chı omium alum

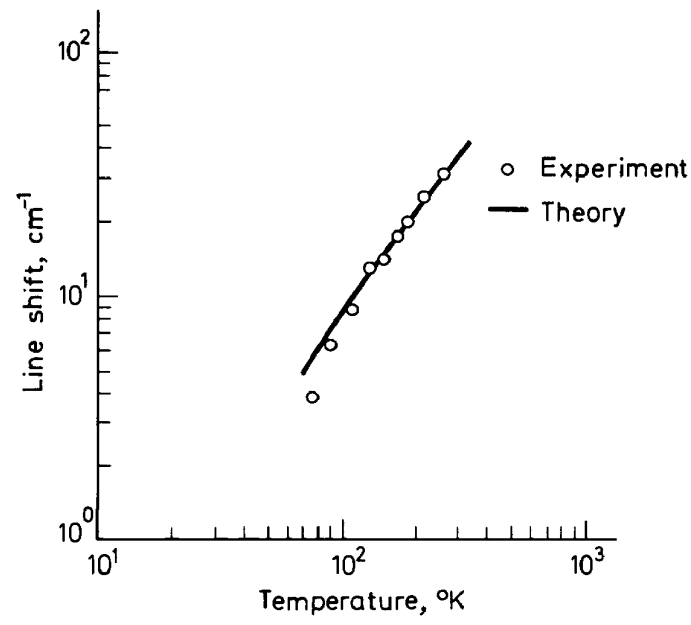

Figure 13. The temperature dependence of the lineshift of the $R_{1}$ line obtained from the same assumption as stated in Figure 12 in thallium chromiun lum 


\section{ABSORPTION LINES IN TRANSITION METAL COMPLEXES}

width of the $B$ lines in chromium alums is several times as great as that of the $R$ lines. This may be due to the lifetime broadening of the $B$ lines which is greater than that of the $R$ lines, and is caused by the direct phonon processes. This results from the fact that the broad $U$ band exists on the lower energy side of the $B$ lines.

The Raman excitation and relaxation processes (1c) may contribute to the linewidth of the $R$ lines as well as the Raman scattering process (3), but it is difficult to discriminate between the contributions of each, because the line broadening due to all the Raman processes shows the same temperature dependence.

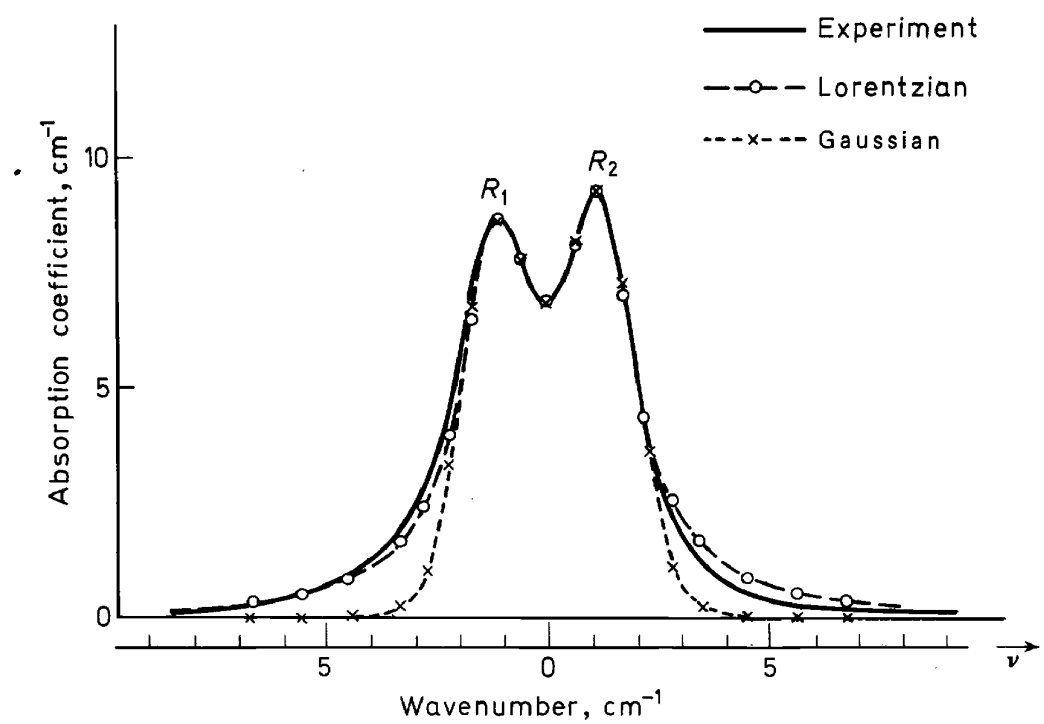

Figure 14. The absorption coefficient curve of the $R$ lines at $77^{\circ} \mathrm{K}$ compared with Lorentzian and Gaussian curves in thallium chromium alum

The line shape of the $R_{1}$ and $R_{2}$ lines at $77^{\circ} \mathrm{K}$ is compared with the Gaussian and Lorentzian shapes as shown in Figure 14. The line shape at $77^{\circ} \mathrm{K}$, like the Lorentzian shape is due to the homogeneous broadening effects. In ruby or $\mathrm{MgO}: \mathrm{Cr}^{3+}$, the linewidth of the $R$ lines at $77^{\circ} \mathrm{K}$ is dominantly caused by heterogeneous strains such as imperfection and dislocation which lead to the Gaussian shape. This difference may be ascribed to the low Debye temperature $\left(\sim 100^{\circ} \mathrm{K}\right)$ of alums and the high Debye temperature $\left(\sim 900^{\circ} \mathrm{K}\right)$ of ruby or $\mathrm{MgO}: \mathrm{Cr}^{3+}$. The specimen-dependent linewidth of the $R$ lines at $4.2^{\circ} \mathrm{K}$ in thallium chromium alum may be caused by a microscopic crystal strain. After all, one $\mathrm{Cr}^{3+}$ ion in the crystal of alum might be considered as an ideal localized system in the sense that magnetic interactions can be disregarded. 


\section{RESEARCHES FOR THE EFFECT OF THE ENVIRONMENT ON THE ABSORPTION LINES}

It is interesting to investigate the effect of the environment on the absorption lines, either electric or magnetic, from the standpoint of the research on phenomena which cannot be dealt with by the ligand field theory. First we investigated spectroscopically the magnetically ordered states, in other words, we studied absorption lines in the antiferromagnetic state of manganous chloride and bromide tetrahydrates ${ }^{37}$ and in the ferrimagnetic state of manganous acetate tetrahydrate ${ }^{38}$ and obtained information about the easy axis, the molecular field and the spin flop phenomenon. Since then, studies of the magnon sidebands (lines) in iron group compounds have been rapidly developed to make one of the most interesting fields of research in solid state physics. It might be also possible to find magnon sidelines in iron group complexes.

At the present time, in order to investigate the electric effect of the environment on the absorption lines, we are studying two absorption lines $\left(R_{1}\right.$ : $14111 \mathrm{~cm}^{-1}, R_{2}: 14131 \mathrm{~cm}^{-1}$ ) of guanidine chromium sulphate hexahydrate $\mathrm{C}\left(\mathrm{NH}_{2}\right)_{3} \mathrm{Cr}\left(\mathrm{SO}_{4}\right)_{2} \cdot 6 \mathrm{H}_{2} \mathrm{O}$ which has a ferroelectric property ${ }^{25,26}$. Crystals of this complex belongs to the trigonal system and the unit cell contains three molecules. $\mathrm{Cr}^{3+}$ ions are surrounded by a distorted octahedron of six water molecules, and one third of the $\mathrm{Cr}^{3+}$ ions are in the $\mathrm{C}_{3 V}$ and two thirds are in the $C_{3}$ site symmetries.

By consideration of the optical anisotropy of the $R$ lines, it can be concluded that the transitions are caused by the electric dipole. This is reasonable because both the two site symmetries of $\mathrm{Cr}^{3+}$ ions have no centre of inversion. But analyses of Zeeman patterns of the $R$ lines are not successful, and then we suppose that the effect of the environment may be significant. The analyses of the $g$ values of the excited states suggest that both the $R_{1}$ and $R_{2}$ lines correspond to the transition from the ground state ${ }^{4} A_{2}$ to the excited state $\bar{E}\left({ }^{2} E\right)$ and each of the $R_{1}$ and $R_{2}$ lines attributes to each of the $C_{3}$ and $C_{3 V}$ symmetry sites in accordance with a conclusion made by MartinBruntière and Couture ${ }^{23}$ and by Camassei and Forster ${ }^{24}$.

An interesting phenomenon was found during the dilution of $\mathrm{Cr}^{3+}$ ions by $\mathrm{Al}^{3+}$ ions ${ }^{25}$; both the $R$ lines split into seven components as shown in Figure 15. Each split component shifts linearly with concentration of $\mathrm{Cr}^{3+}$ ions and splittings between neighbouring components are nearly constant at $6 \mathrm{~cm}^{-1}$. Absorption intensity of each component varies with concentration and has a maximum at a certain concentration.

To explain the phenomenon, one may consider a model which consists of a central $\left[\mathrm{Cr}\left(\mathrm{H}_{2} \mathrm{O}\right)_{6}\right]^{3+}$ ion and six nearest neighbours of $\left[\mathrm{M}\left(\mathrm{H}_{2} \mathrm{O}\right)_{6}\right]^{3+}$ ions $(\mathrm{M}$ is $\mathrm{Cr}$ or $\mathrm{Al})$, equidistant from the central ion in the plane normal to the trigonal axis $C_{3}$, as shown in Figure 16. The electric field strength at the central ion produced by the nearest neighbour $\left[\mathrm{Cr}\left(\mathrm{H}_{2} \mathrm{O}\right)_{6}\right]^{3+}$ ion must be different from that produced by a nearest neighbour $\left[\mathrm{Al}\left(\mathrm{H}_{2} \mathrm{O}\right)_{6}\right]^{3+}$ ion and then seven different arrangements exist depending on the number of $\left[\mathrm{Cr}\left(\mathrm{H}_{2} \mathrm{O}\right)_{6}\right]^{3+}$ ions in the six nearest neighbours $(n=0,1,2, \ldots 6)$.

The absorption intensity of each component might be proportional to the probability for existence of each arrangement in the crystal. Concentration 


\section{ABSORPTION LINES IN TRANSITION METAL COMPLEXES}

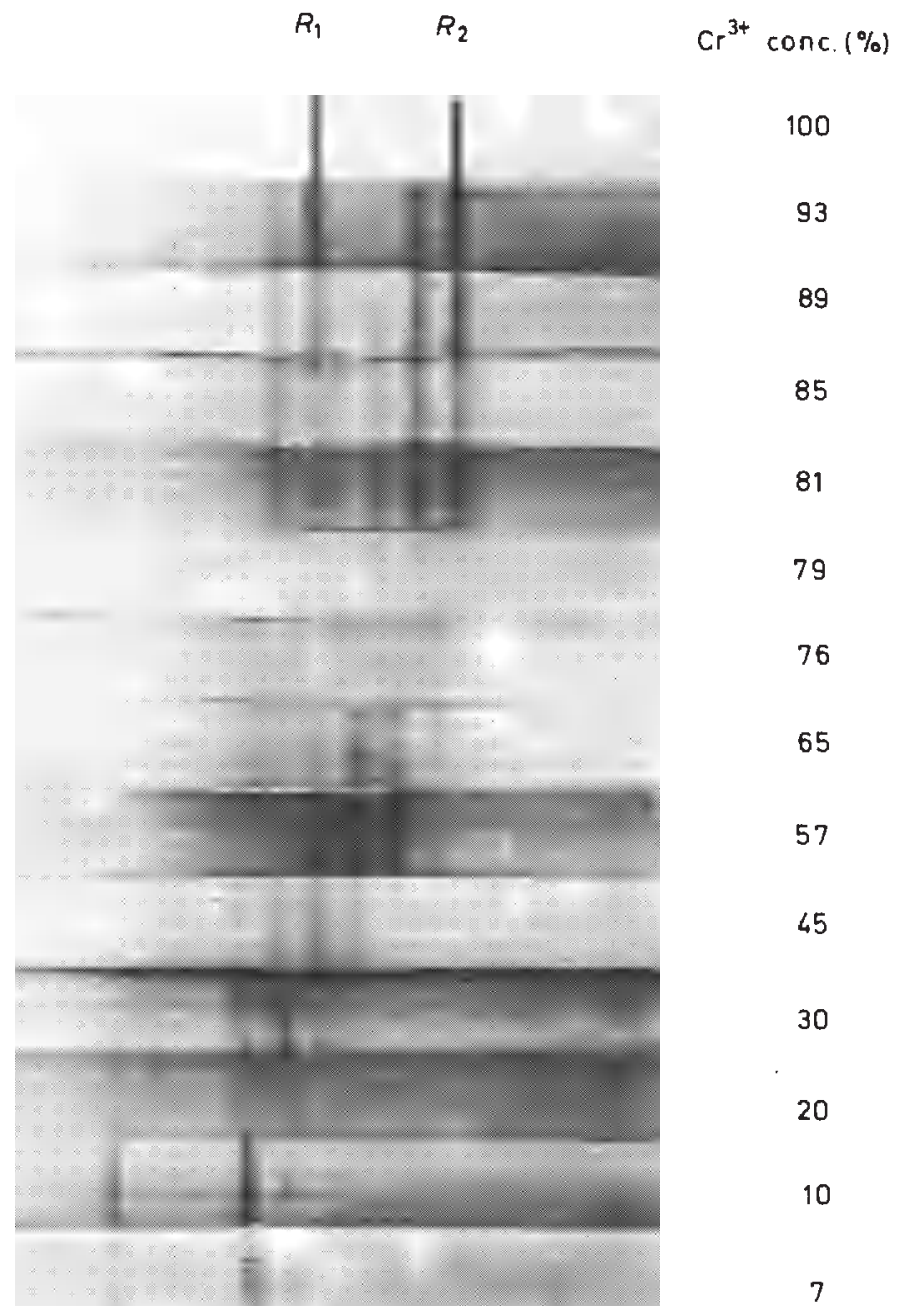

Figure 15. Splitting of the $R$ lines under dilution of $\mathrm{Cr}^{3+}$ ions by $\mathrm{Al}^{3+}$ ions in guanidine chromium sulphate hexahydrate 


\section{IKUJI TSUJIKAWA}

dependence of absorption intensity is well accounted for if probabilities of these seven different arrangements are taken, under the assumption of the random distribution of $\mathrm{Cr}^{3+}$ ions over the crystal without preference to occupy the $C_{3}$ or $C_{3 V}$ symmetry sites. The splitting of $6 \mathrm{~cm}^{-1}$ between two neighbouring components results from the difference between the electric field strengths on the central ions for the two adjacent arrangements in Figure 16.
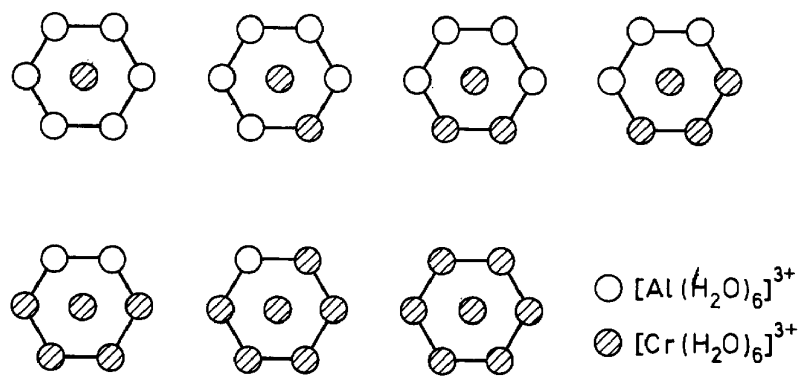

Figure 16. Model of arrangements of nearest neighbours of $\left[\mathrm{M}\left(\mathrm{H}_{2} \mathrm{O}\right)_{6}\right]^{3+}$ ions around the central $\left[\mathrm{Cr}\left(\mathrm{H}_{2} \mathrm{O}\right)_{6}\right]^{3+}$ ion in guanidine chromium sulphate hexahydrate. Open and shaded circles represent $\left[\mathrm{Al}\left(\mathrm{H}_{2} \mathrm{O}\right)_{6}\right]^{3+}$ and $\left[\mathrm{Cr}\left(\mathrm{H}_{2} \mathrm{O}\right)_{6}\right]^{3+}$ ions respectively

The shift of components linearly dependent on concentration may be explained by considering that a part of the electric field for $\mathrm{Cr}^{3+}$ sites results from the concentration dependent spontaneous polarization of the whole crystal.

I give sincere thanks to the Organizing Committee of the Conference for affording me the opportunity of presenting a plenary lecture in this honourable meeting and also to my collaborators in our laboratory for their eager and constant efforts.

\section{REFERENCES}

Y. Tanabe and S. Sugano, J. Phys. Soc Japan, 9, 753, 766 (1954).

L. E. Orgel, J. Chem. Phys. 23, 1004 (1954).

${ }^{3}$ S. Sugano and Y. Tanabe, J. Phys. Soc. Japan, 13, 880 (1958).

4 S. Sugano and I. Tsujikawa, J. Phys. Soc. Japan, 13, 899 (1958).

5 M. H. L. Pryce and W. A. Runciman, Dis. Faraday Soc. 26, 34 (1958).

6 S. Sugano, Proc. Theor. Phys. Suppl. 14, 66 (1960).

7 S. Sakatsume and I. Tsujikawa, J. Phys. Soc. Japan, 19, 1080 (1964).

8 W. Kaiser, S. Sugano and D. L. Wood, Phys. Rev. Letters, 6, 535 (1961).

9 S. Sugano and M. Peter, Phys. Rev., 122, 381 (1961).

$10 \mathrm{~J}$. Brossel and J. Margerie, Proceedings of the First International Conference on Paramagnetic Resonance, Jerusalem (July 1962).

11 J. Margerie, C.R. Acid. Sci., Paris, 255, 1598 (1962).

12 S. Sugano, A. L. Schawlow and F. Varsanyi, Phys. Rev., 120, 2045 (1960).

13 A. L. Schawlow, A. H. Piksis and S. Sugano, Phys. Rev. 122, 1469 (1961).

14 K. Schnetzler, Ann. Phys. Lpz. 10, 373 (1931). 


\section{ABSORPTION LINES IN TRANSITION METAL COMPLEXES}

15 F. H. Spedding and G. C. Nutting, J. Chem. Phys. 3, 369 (1935).

16 D. L. Kraus and G. C. Nutting, J. Chem. Phys. 9,133 (1941).

17 L. Couture, P. Jacquinot and I. Tsujikawa, Proceedings of the Conference on Low Temperature Physics, Paris (October 1955).

18 N. Koshizuka, T. Ban, I. Tsujikawa and S. Sugano, J. Phys. Soc. Japan, 25, 921 (1968).

19 Van Vleck, J. Chem. Phys. 8, 787 (1940).

${ }^{20}$ I. Tsujikawa, M. Tamatani, N. Koshizuka, Y. Uesaka and T. Ban, XIth ICCC, Nikko (September 1967).

21 N. Koshizuka, T. Ban and I. Tsujikawa, J. Phys. Soc. Japan, 30, 470 (1971)

22 Y. Uesaka, T. Ban and I. Tsujikawa, J. Phys. Soc. Japan, 30, 228 (1971)

${ }^{23}$ F. Martin-Bruntière and L. Couture, C.R. Acad. Sci., Paris, 256, 5327 (1963).

${ }^{24}$ F. D. Camassei and L. S. Forster, J. Molec. Spectr. 31, 129 (1969).

25 M. Tamatani, T. Ban and I. Tsujikawa, J. Phys. Soc. Japan, 24, 1183 (1968).

26 M. Tamatani, T. Ban and I. Tsujikawa, J. Phys. Soc. Japan, 30, 481 (1971).

27 K. Aoyagi, A. Misu and S. Sugano, J. Phys. Soc. Japan, 18, 1448 (1963).

${ }^{28}$ B. P. Zakharchenya, A. I. Sibilv, L. M. Kanskaya and A. Y. Ryskin, Fiz. Tverdogo Tela, 3, $3531(1960)$.

29 I. Tsujikawa and L. Couture, C.R. Acad. Sci., Paris, 250, 2013 (1960).

${ }^{30}$ I. Tsujikawa, J. Phys. Soc. Japan, 18, 1391 (1963).

31 A. Le Paillier-Malecot, C.R. Acad. Sci., Paris, 260, 1142 (1965).

32 R. Pappalardo, Phil. Mag. 4, 219 (1959).

33 M. H. L. Pryce, Phil. Mag. 10, 477 (1964).

34 N. Pelletier-Allard, C.R. Acad. Sci., Paris, 259, 2999(1964).

35 J. Ferguson, J. Chem. Phys. 39, 116(1963).

36 B. di Bartolo, Optical Interactions in Solids. Wiley: New York (1968).

37 I. Tsujikawa and E. Kanda, J. Phys. Soc. Japan, 18, 1382 (1963).

38 I. Tsujikawa, J.'Phys. Soc. Japan, 18, 1407 (1963). 